\title{
Some weighted geometric operators with SVTrN-numbers and their application to multi-criteria decision making problems
}

\author{
Irfan Deli, Yusuf Şubaş \\ Muallim Rifat Faculty of Education, \\ 7 Aralık University, 79000 Kilis, Turkey \\ irfandeli@kilis.edu.tr, ysubas@kilis.edu.tr
}

March 3, 2015

\begin{abstract}
The single valued triangular neutrosophic number (SVTrN-number) is simply an ordinary number whose precise value is somewhat uncertain from a philosophical point of view, which is a generalization of triangular fuzzy numbers and triangular intuitionistic fuzzy numbers. Also, SVTrN-number may express more abundant and flexible information as compared with the triangular fuzzy numbers and triangular intuitionistic fuzzy numbers. This article introduces an approach to handle multi-criteria decision making (MCDM) problems under the SVTrN-numbers. Therefore, we first proposed some new geometric operator is called SVTrN weighted geometric operator, SVTrN ordered weighted geometric operator, SVTrN ordered hybrid weighted geometric operator. Also we studied some desirable properties of the geometric operators. And then, an approach based on the SVTrN ordered hybrid weighted geometric operator is developed to solve multi-criteria decision making problems with SVTrN-number. Finally, a numerical example is used to demonstrate how to apply the proposed approach.
\end{abstract}

Keyword 0.1 Neutrosophic set, single valued neutrosophic numbers, triangular neutrosophic numbers, geometric operators, decision making.

\section{Introduction}

Zadeh [44] proposed the notation of fuzzy set $\mathrm{X}$ on a fixed set $\mathrm{E}$ characterized by a membership function denoted by $\mu_{X}$ such that $\mu_{X}: E \rightarrow[0,1]$ which are the powerful tools to deal with imperfect and imprecise information. Then, by adding non-membership function to fuzzy sets, Atanassov [1] presented the notation of intuitionistic fuzzy set $\mathrm{K}$ on a fixed set $\mathrm{E}$ characterized by a membership function $\mu_{K}: E \rightarrow[0,1]$ and a non-membership function $\gamma_{K}: E \rightarrow[0,1]$ such that such that $0 \leq \mu_{K}(x)+\gamma_{K}(x) \leq 1$ for any $x \in E$, which is a generalization of fuzzy set [44]. By Smarandache [24], intuitionistic fuzzy set was extended to develop the notation of neutrosophic set $\mathrm{A}$ on a fixed set $\mathrm{E}$ characterized by a truth-membership function $T_{A}$, a indeterminacy-membership function $I_{A}$ and a falsity-membership function $F_{A}$ such that $T_{A}(x), I_{A}(x)$, $\left.F_{A}(x) \in\right]^{-} 0,1\left[^{+}\right.$which is a generalization of fuzzy set and intuitionistic fuzzy set. The neutrosophic sets may express more abundant and flexible information as compared with the fuzzy sets and intuitionistic fuzzy sets. Recently, neutrosophic sets have been researched by many scholars in different fields. For example; on neutrosophic similarity clustering $[4,6,7,39,40,43]$, on multi-criteria decision making problems [38, 41] etc. Also the notations such as fuzzy sets, intuitionistic fuzzy sets and neutrosophic sets has been applied to some different fields in $[3,5,8,9,11,12,13,18,25,28,36,42]$.

Aggregation operators, which is an important research topic in decision-making theory, have been researched by many scholars such as; intuitionistic fuzzy sets [14, 19, 21, 26], intuitionistic fuzzy numbers $[14,15,17,22,27,29,30,31,32,33,35]$, neutrosophic sets [2, 20, 23], neutrosophic number [41], and so on. Especially, Xu and Yager [26], introduced some new geometric aggregation operators, is called intuitionistic fuzzy weighted geometric operator, intuitionistic fuzzy ordered weighted geometric operator, and intuitionistic fuzzy hybrid geometric operator. Also, Wu and Cao [32] presented some geometric aggregation operators with intuitionistic trapezoidal fuzzy numbers. 
Since neutrosophic numbers [10] are a special case of neutrosophic sets, the neutrosophic numbers are importance for neutrosophic multi criteria decision making (MCDM) problems. As a generalization of fuzzy numbers and intuitionistic fuzzy number, a neutrosophic number seems to suitably describe an ill-known quantity. To the our knowledge, existing approaches are not suitable for dealing with MCDM problems under SVTrN-numbers. Therefore, the remainder of this paper is organized as follows: In section 2, some basic definitions of fuzzy sets, intuitionistic fuzzy sets, neutrosophic sets, single valued neutrosophic sets and single valued triangular neutrosophic number are briefly reviewed. In section 3, some new geometric operator is called SVTrN weighted geometric operator, SVTrN ordered weighted geometric operator, SVTrN ordered hybrid weighted geometric operator are defined(adapted from [41, 14]. In section 4, an approach based on the SVTrN ordered hybrid weighted geometric operator is developed to solve multi-criteria decision making problems with single valued triangular neutrosophic number is developed. In section 5 , a numerical example is given to demonstrate how to apply the proposed approach. In section 7, the study is concluded.

\section{Preliminary}

In this section, we recall some basic notions of fuzzy sets [44], intuitionistic fuzzy sets [1], intuitionistic fuzzy numbers [14] and neutrosophic sets [24]. For more details, the reader could refer to [1, 14, 24, 25, 44]. From now on we use $I_{n}=\{1,2, \ldots, n\}$ and $I_{m}=\{1,2, \ldots, m\}$ as an index set for $n \in N$ and $m \in N$, respectively.

Definition 2.1 [44] Let $E$ be a universe. Then a fuzzy set $X$ over $E$ is a function defined as follows:

$$
X=\left\{\left(\mu_{X}(x) / x\right): x \in E\right\}
$$

where $\mu_{X}: E \rightarrow[0.1]$.

Here, $\mu_{X}$ called membership function of $X$, and the value $\mu_{X}(x)$ is called the grade of membership of $x \in E$. The value represents the degree of $x$ belonging to the fuzzy set $X$.

Definition 2.2 [1] Let $E$ be a universe. An intuitionistic fuzzy set $K$ on $E$ can be defined as follows:

$$
K=\left\{<x, \mu_{K}(x), \gamma_{K}(x)>: x \in E\right\}
$$

where, $\mu_{K}: E \rightarrow[0,1]$ and $\gamma_{K}: E \rightarrow[0,1]$ such that $0 \leq \mu_{K}(x)+\gamma_{K}(x) \leq 1$ for any $x \in E$.

Here, $\mu_{K}(x)$ and $\gamma_{K}(x)$ is the degree of membership and degree of non-membership of the element $x$, respectively.

Definition 2.3 [24] Let $E$ be a universe. A neutrosophic sets(NS) $A$ in $E$ is characterized by a truthmembership function $T_{A}$, a indeterminacy-membership function $I_{A}$ and a falsity-membership function $F_{A}$. $T_{A}(x) ; I_{A}(x)$ and $F_{A}(x)$ are real standard elements of $[0,1]$. It can be written as

$$
A=\left\{<x,\left(T_{A}(x), I_{A}(x), F_{A}(x)\right)>: x \in E, T_{A}(x), I_{A}(x), F_{A}(x) \in\right]^{-} 0,1\left[^{+}\right\} .
$$

There is no restriction on the sum of $T_{A}(x) ; I_{A}(x)$ and $F_{A}(x)$, so $0^{-} \leq T_{A}(x)+I_{A}(x)+F_{A}(x) \leq 3^{+}$.

Definition 2.4 [28] Let $E$ be a universe. A single valued neutrosophic sets(SVNS) $A$, which can be used in real scientific and engineering applications, in $E$ is characterized by a truth-membership function $T_{A}$, a indeterminacy-membership function $I_{A}$ and a falsity-membership function $F_{A} . T_{A}(x) ; I_{A}(x)$ and $F_{A}(x)$ are real standard elements of $[0,1]$. It can be written as

$$
A=\left\{<x,\left(T_{A}(x), I_{A}(x), F_{A}(x)\right)>: x \in E, T_{A}(x), I_{A}(x), F_{A}(x) \in[0,1]\right\} .
$$

There is no restriction on the sum of $T_{A}(x) ; I_{A}(x)$ and $F_{A}(x)$, so $0 \leq T_{A}(x)+I_{A}(x)+F_{A}(x) \leq 3$. 
Definition 2.5 [10] Let $w_{\tilde{a}}, u_{\tilde{a}}, y_{\tilde{a}} \in[0,1]$ and $a_{1}, b_{1}, c_{1} \in R$ such that $a_{1} \leq b_{1} \leq c_{1}$. Then, a single valued triangular neutrosophic number (SVTrN-number)

$$
\tilde{a}=\left\langle\left(a_{1}, b_{1}, c_{1}\right) ; w_{\tilde{a}}, u_{\tilde{a}}, y_{\tilde{a}}\right\rangle
$$

is a special neutrosophic set on the real number set $R$, whose truth-membership indeterminacy-membership and falsity-membership functions are defined as follows:

$$
\mu_{\tilde{a}}(x)= \begin{cases}\left(x-a_{1}\right) w_{\tilde{a}} /\left(b_{1}-a_{1}\right) & \left(a_{1} \leq x<b_{1}\right) \\ w_{\tilde{a}} & \left(x=b_{1}\right) \\ \left(c_{1}-x\right) w_{\tilde{a}} /\left(c_{1}-b_{1}\right) & \left(b_{1}<x \leq c_{1}\right) \\ 0 & \text { otherwise }\end{cases}
$$

and

$$
\begin{gathered}
\nu_{\tilde{a}}(x)= \begin{cases}\left(b_{1}-x+u_{\tilde{a}}\left(x-a_{1}\right)\right) /\left(b_{1}-a_{1}\right) & \left(a_{1} \leq x<b_{1}\right) \\
u_{\tilde{a}} & \left(x=b_{1}\right) \\
\left(x-b_{1}+u_{\tilde{a}}\left(c_{1}-x\right)\right) /\left(c_{1}-b_{1}\right) & \left(b_{1}<x \leq c_{1}\right) \\
1 & \text { otherwise, }\end{cases} \\
\lambda_{\tilde{a}}(x)= \begin{cases}\left(b_{1}-x+y_{\tilde{a}}\left(x-a_{1}\right)\right) /\left(b_{1}-a_{1}\right) & \left(a_{1} \leq x<b_{1}\right) \\
y_{\tilde{a}} & \left(x=b_{1}\right) \\
\left(x-b_{1}+y_{\tilde{a}}\left(c_{1}-x\right)\right) /\left(c_{1}-b_{1}\right) & \left(b_{1}<x \leq c_{1}\right) \\
1 & \text { otherwise, }\end{cases}
\end{gathered}
$$

respectively.

If $a_{1} \geq 0$ and at least $c_{1}>0$ then $\tilde{a}=\left\langle\left(a_{1}, b_{1}, c_{1}\right) ; w_{\tilde{a}}, u_{\tilde{a}}, y_{\tilde{a}}\right\rangle$ is called a positive SVTrN-numbers, denoted by $\tilde{a}>0$. Likewise, if $c_{1} \leq 0$ and at least $a_{1}<0$, then $\tilde{a}=\left\langle\left(a_{1}, b_{1}, c_{1}\right) ; w_{\tilde{a}}, u_{\tilde{a}}, y_{\tilde{a}}\right\rangle$ is called a negative SVTrN-numbers, denoted by $\tilde{a}<0$. A SVTrN-numbers $\tilde{a}=\left\langle\left(a_{1}, b_{1}, c_{1}\right) ; w_{\tilde{a}}, u_{\tilde{a}}, y_{\tilde{a}}\right\rangle$ may express an ill-known quantity about a, which is approximately equal to a.

Note that the set of all SVTrN-numbers on $R$ will be denoted by $\Delta$.

Definition 2.6 [10] Let $\tilde{a}=\left\langle\left(a_{1}, b_{1}, c_{1}\right) ; w_{\tilde{a}}, u_{\tilde{a}}, y_{\tilde{a}}\right\rangle, \tilde{b}=\left\langle\left(a_{2}, b_{2}, c_{2}\right) ; w_{\tilde{b}}, u_{\tilde{b}}, y_{\tilde{b}}\right\rangle \in \Delta$ and $\gamma \neq 0$ be any real number. Then,

1. $\tilde{a}+\tilde{b}=\left\langle\left(a_{1}+a_{2}, b_{1}+b_{2}, c_{1}+c_{2}\right) ; w_{\tilde{a}} \wedge w_{\tilde{b}}, u_{\tilde{a}} \vee u_{\tilde{b}}, y_{\tilde{a}} \vee y_{\tilde{b}}\right\rangle$

2. $\tilde{a}-\tilde{b}=\left\langle\left(a_{1}-c_{2}, b_{1}-b_{2}, c_{1}-a_{2}\right) ; w_{\tilde{a}} \wedge w_{\tilde{b}}, u_{\tilde{a}} \vee u_{\tilde{b}}, y_{\tilde{b}} \vee y_{\tilde{b}}\right\rangle$

3. $\tilde{a} \tilde{b}= \begin{cases}\left\langle\left(a_{1} a_{2}, b_{1} b_{2}, c_{1} c_{2} ; w_{\tilde{a}} \wedge w_{\tilde{b}}, u_{\tilde{a}} \vee u_{\tilde{b}}, y_{\tilde{a}} \vee y_{\tilde{b}}\right\rangle\right. & \left(c_{1}>0, c_{2}>0\right) \\ \left\langle\left(a_{1} c_{2}, b_{1} b_{2}, c_{1} a_{2}\right) ; w_{\tilde{a}} \wedge w_{\tilde{b}}, u_{\tilde{a}} \vee u_{\tilde{b}}, y_{\tilde{a}} \vee y_{\tilde{b}}\right\rangle & \left(c_{1}<0, c_{2}>0\right) \\ \left\langle\left(c_{1} c_{2}, b_{1} b_{2}, a_{1} a_{2}\right) ; w_{\tilde{a}} \wedge w_{\tilde{b}}, u_{\tilde{a}} \vee u_{\tilde{b}}, y_{\tilde{a}} \vee y_{\tilde{b}}\right\rangle & \left(c_{1}<0, c_{2}<0\right)\end{cases}$

4. $\tilde{a} / \tilde{b}= \begin{cases}\left\langle\left(a_{1} / c_{2}, b_{1} / b_{2}, c_{1} / a_{2}\right) ; w_{\tilde{a}} \wedge w_{\tilde{b}}, u_{\tilde{a}} \vee u_{\tilde{b}}, y_{\tilde{a}} \vee y_{\tilde{b}}\right\rangle & \left(c_{1}>0, c_{2}>0\right) \\ \left\langle\left(c_{1} / c_{2}, b_{1} / b_{2}, a_{1} / a_{2}\right) ; w_{\tilde{a}} \wedge w_{\tilde{b}}, u_{\tilde{a}} \vee u_{\tilde{b}}, y_{\tilde{a}} \vee y_{\tilde{b}}\right\rangle & \left(c_{1}<0, c_{2}>0\right) \\ \left\langle\left(c_{1} / a_{2}, b_{1} / b_{2}, a_{1} / c_{2}\right) ; w_{\tilde{a}} \wedge w_{\tilde{b}}, u_{\tilde{a}} \vee u_{\tilde{b}}, y_{\tilde{a}} \vee y_{\tilde{b}}\right\rangle & \left(c_{1}<0, c_{2}<0\right)\end{cases}$

5. $\gamma \tilde{a}= \begin{cases}\left\langle\left(\gamma a_{1}, \gamma b_{1}, \gamma c_{1}\right) ; w_{\tilde{a}}, u_{\tilde{a}}, y_{\tilde{a}}\right\rangle & (\gamma>0) \\ \left\langle\left(\gamma c_{1}, \gamma b_{1}, \gamma a_{1}\right) ; w_{\tilde{a}}, u_{\tilde{a}}, y_{\tilde{a}}\right\rangle & (\gamma<0)\end{cases}$

6. $\tilde{a}^{\gamma}= \begin{cases}\left\langle\left(a_{1}^{\gamma}, b_{1}^{\gamma}, c_{1}^{\gamma}\right) ; w_{\tilde{a}}, u_{\tilde{a}}, y_{\tilde{a}}\right\rangle & (\gamma>0) \\ \left\langle\left(c_{1}^{\gamma}, b_{1}^{\gamma}, a_{1}^{\gamma}\right) ; w_{\tilde{a}}, u_{\tilde{a}}, y_{\tilde{a}}\right\rangle & (\gamma<0)\end{cases}$

Likewise, it is easily proven that the results obtained by multiplication and division of two SVTrNnumbers are not always SVTrN-numbers. However, we often use SVTrN-numbers to express these computational results approximately.

Example 2.7 Let $\tilde{a}=\langle(4,5,6) ; 0.7,0.5,0.3\rangle$ and $\tilde{b}=\langle(2,3,4) ; 0.6,0.1,0.4\rangle$ be two SVTrN-numbers then, 
1. $\tilde{a}+\tilde{b}=\langle(6,8,10) ; 0.6,0.5,0.4\rangle$

2. $\tilde{a}-\tilde{b}=\langle(0,2,4) ; 0.6,0.5,0.4\rangle$

3. $\tilde{a} \tilde{b}=\langle(8,15,24) ; 0.6,0.5,0.4\rangle$

4. $\tilde{a} / \tilde{b}=\left\langle\left(1, \frac{5}{3}, 3\right) ; 0.6,0.5,0.4\right\rangle$

5. $2 \tilde{a}=\langle(8,10,12) ; 0.7,0.5,0.3\rangle$

6. $\tilde{b}^{2}=\langle(4,9,16) ; 0.6,0.1,0.4\rangle$

Definition 2.8 [10] We defined a method to compare any two SVTrN-numbers which is based on the score function and the accuracy function. Let $\tilde{a}=\left\langle(a, b, c) ; w_{\tilde{a}}, u_{\tilde{a}}, y_{\tilde{a}}\right\rangle \in \Delta$, then

$$
S(\tilde{a})=\frac{1}{8}[a+b+c] \times\left(2+\mu_{\tilde{a}}-\nu_{\tilde{a}}-\gamma_{\tilde{a}}\right)
$$

and

$$
A(\tilde{a})=\frac{1}{8}[a+b+c] \times\left(2+\mu_{\tilde{a}}-\nu_{\tilde{a}}+\gamma_{\tilde{a}}\right)
$$

is called the score and accuracy degrees of $\tilde{a}$, respectively.

Definition 2.9 [10] Let $\tilde{a}_{1}, \tilde{a}_{2} \in \Delta$. Then,

1. If $S\left(\tilde{a}_{1}\right)<S\left(\tilde{a}_{2}\right)$, then $\tilde{a}_{1}$ is smaller than $\tilde{a}_{2}$, denoted by $\tilde{a}_{1}<\tilde{a}_{2}$

2. If $S\left(\tilde{a}_{1}\right)>S\left(\tilde{a}_{2}\right)$, then $\tilde{a}_{1}$ is bigger than $\tilde{a}_{2}$, denoted by $\tilde{a}_{1}>\tilde{a}_{2}$

3. If $S\left(\tilde{a}_{1}\right)=S\left(\tilde{a}_{2}\right)$;

(a) If $A\left(\tilde{a}_{1}\right)<A\left(\tilde{a}_{2}\right)$, then $\tilde{a}_{1}$ is smaller than $\tilde{a}_{2}$, denoted by $\tilde{a}_{1}<\tilde{a}_{2}$

(b) If $A\left(\tilde{a}_{1}\right)>A\left(\tilde{a}_{2}\right)$, then $\tilde{a}_{1}$ is bigger than $\tilde{a}_{2}$, denoted by $\tilde{a}_{1}>\tilde{a}_{2}$

(c) If $A\left(\tilde{a}_{1}\right)=A\left(\tilde{a}_{2}\right)$, then $\tilde{a}_{1}$ and $\tilde{a}_{2}$ are the same, denoted by $\tilde{a}_{1}=\tilde{a}_{2}$

\section{Geometric operators of the SVTrN-number}

In this section, three SVTrN weighted geometric operator of SVTrN-numbers is called SVTrN weighted geometric operator, SVTrN ordered weighted geometric operator, SVTrN ordered hybrid weighted geometric operator is given. Some of it is quoted from application in $[10,14,15,16,17,18,26,31,32,37]$.

Definition 3.1 Let $\tilde{a}_{j}=\left\langle\left(a_{j}, b_{j}, c_{j}\right) ; w_{\tilde{a}_{j}}, u_{\tilde{a}_{j}}, y_{\tilde{a}_{j}}\right\rangle \in \Delta\left(j \in I_{n}\right)$. Then SVTrN weighted geometric operator, denoted by $G_{g o}$, is defined as;

$$
G_{g o}: \Delta^{n} \rightarrow \Delta, \quad G_{g o}\left(\tilde{a}_{1}, \tilde{a}_{2}, \ldots, \tilde{a}_{n}\right)=\prod_{i=1}^{n} \tilde{a}_{i}^{w_{i}}
$$

where, $w=\left(w_{1}, w_{2}, \ldots, w_{n}\right)^{T}$ is a weight vector associated with the $G_{g o}$ operator, for every $j \in I_{n}$ such that, $w_{j} \in[0,1]$ and $\sum_{j=1}^{n} w_{j}=1$.

Theorem 3.2 Let $\tilde{a}_{j}=\left\langle\left(a_{j}, b_{j}, c_{j}\right) ; w_{\tilde{a}_{j}}, u_{\tilde{a}_{j}}, y_{\tilde{a}_{j}}\right\rangle \in \Gamma(j \in I), w=\left(w_{1}, w_{2}, \ldots, w_{n}\right)^{T}$ be a weight vector of $\tilde{a}_{j}$, for every $j \in I_{n}$ such that $w_{j} \in[0,1]$ and $\sum_{j=1}^{n} w_{j}=1$. Then, their aggregated value by using $G_{g o}$ operator is also a $S V T r N$-number and

$$
G_{g o}\left(\tilde{a}_{1}, \tilde{a}_{2}, \ldots, \tilde{a}_{n}\right)=\left\langle\left(\prod_{j=1}^{n} a_{j}^{w_{j}}, \prod_{j=1}^{n} b_{j}^{w_{j}}, \prod_{j=1}^{n} c_{j}^{w_{j}}\right) ; \bigwedge_{j=1}^{n} w_{\tilde{a}_{j}}, \bigvee_{j=1}^{n} u_{\tilde{a}_{j}}, \bigvee_{j=1}^{n} y_{\tilde{a}_{j}}\right\rangle
$$


Proof The proof can be made by using mathematical induction on $n$ as; Assume that,

$$
\tilde{a}_{1}=\left\langle\left(a_{1}, b_{1}, c_{1}\right) ; w_{\tilde{a}_{1}}, u_{\tilde{a}_{1}}, y_{\tilde{a}_{1}}\right\rangle
$$

and

$$
\tilde{a}_{2}=\left\langle\left(a_{2}, b_{2}, c_{2}\right) ; w_{\tilde{a}_{2}}, u_{\tilde{a}_{2}}, y_{\tilde{a}_{2}}\right\rangle
$$

be two SVTrN-numbers then, for $n=2$, we have

$$
G_{\text {ogo }}\left(\tilde{a}_{1}, \tilde{a}_{2}\right)=\left\langle\left(\prod_{j=1}^{2} a_{j}^{w_{j}}, \prod_{j=1}^{2} b_{j}^{w_{j}}, \prod_{j=1}^{2} c_{j}^{w_{j}}\right) ; \bigwedge_{j=1}^{2} w_{\tilde{a}_{j}}, \bigvee_{j=1}^{2} u_{\tilde{a}_{j}}, \bigvee_{j=1}^{2} y_{\tilde{a}_{j}}\right\rangle
$$

If holds for $n=k$, that is

$$
G_{\text {ogo }}\left(\tilde{a}_{1}, \tilde{a}_{2}, \ldots, \tilde{a}_{k}\right)=\left\langle\left(\prod_{j=1}^{k} a_{j}^{w_{j}}, \prod_{j=1}^{k} b_{j}^{w_{j}}, \prod_{j=1}^{k} c_{j}^{w_{j}}\right) ; \bigwedge_{j=1}^{k} w_{\tilde{a}_{j}}, \bigvee_{j=1}^{k} u_{\tilde{a}_{j}}, \bigvee_{j=1}^{k} y_{\tilde{a}_{j}}\right\rangle
$$

then, when $n=k+1$, by the operational laws in Definition 2.6, I have

$$
\begin{aligned}
G_{\text {ogo }}\left(\tilde{a}_{1}, \tilde{a}_{2}, \ldots, \tilde{a}_{k}, \tilde{a}_{k+1}\right)=\left\langle\left(\prod_{j=1}^{k} a_{j}^{w_{j}}, \prod_{j=1}^{k} b_{j}^{w_{j}}, \prod_{j=1}^{k} c_{j}^{w_{j}}\right) ; \bigwedge_{j=1}^{k} w_{\tilde{a}_{j}}, \bigvee_{j=1}^{k} u_{\tilde{a}_{j}}, \bigvee_{j=1}^{k} y_{\tilde{a}_{j}}\right\rangle \\
\times\left\langle\left(w_{k+1} a_{k+1}, w_{k+1} b_{k+1}, w_{k+1} c_{k+1}\right) ; w_{\tilde{a}_{k+1}}, u_{\tilde{a}_{k+1}}, y_{\tilde{a}_{k+1}}\right\rangle \\
=\left\langle\left(\prod_{j=1}^{k+1} a_{j}^{w_{j}}, \prod_{j=1}^{k+1} b_{j}^{w_{j}}, \prod_{j=1}^{k+1} c_{j}^{w_{j}}\right) ; \bigwedge_{j=1}^{k+1} w_{\tilde{a}_{j}}, \bigvee_{j=1}^{k+1} u_{\tilde{a}_{j}}, \bigvee_{j=1}^{k+1} y_{\tilde{a}_{j}}\right\rangle
\end{aligned}
$$

therefore proof is valid.

Definition 3.3 Let $\tilde{a}_{j}=\left\langle\left(a_{j}, b_{j}, c_{j}\right) ; w_{\tilde{a}_{j}}, u_{\tilde{a}_{j}}, y_{\tilde{a}_{j}}\right\rangle \in \Delta\left(j \in I_{n}\right)$. Then SVTrN ordered weighted geometric operator denoted by $G_{\text {ogo }}$, is defined as;

$$
G_{\text {ogo }}: \Delta^{n} \rightarrow \Delta, G_{\text {ogo }}\left(\tilde{a}_{1}, \tilde{a}_{2}, \ldots, \tilde{a}_{n}\right)=\prod_{k=1}^{n} \tilde{b}_{k}^{w_{k}}
$$

where $w=\left(w_{1}, w_{2}, \ldots, w_{n}\right)^{T}$ is a weight vector associated with the mapping $G_{\text {ogo }}$, which satisfies the normalized conditions: $w_{k} \in[0,1]$ and $\sum_{k=1}^{n} w_{k}=1 ; \tilde{b}_{k}=\left\langle\left(a_{k}, b_{k}, c_{k}\right) ; w_{\tilde{a}_{k}}, u_{\tilde{a}_{k}}, y_{\tilde{a}_{k}}\right\rangle$ is the k-th largest of the $n$ $S V T r N$-numbers $\tilde{a}_{j}\left(j \in I_{n}\right)$ which is determined through using ranking method in Definition 2.8.

It is not difficult to follows from Definition 3.3 that

$$
\begin{aligned}
G_{\text {ogo }}\left(\tilde{a}_{1}, \tilde{a}_{2}, \ldots, \tilde{a}_{n}\right) & =\prod_{k=1}^{n} \tilde{b}_{k}^{w_{k}} \\
& =\prod_{k=1}^{n=1}\left(\left\langle\left(a_{k}, b_{k}, c_{k}\right) ; w_{\tilde{a}_{k}}, u_{\tilde{a}_{k}}, y_{\tilde{a}_{k}}\right\rangle\right)^{w_{k}} \\
& =\prod_{k=1}^{n}\left\langle\left(a_{k}^{w_{k}}, b_{k}^{w_{k}}, c_{k}^{w_{k}}\right) ; w_{\tilde{a}_{k}}, u_{\tilde{a}_{k}}, y_{\tilde{a}_{k}}\right\rangle \\
& =\left\langle\left(\prod_{k=1}^{n} a_{k}^{w_{k}}, \prod_{k=1}^{n} b_{k}^{w_{k}}, \prod_{k=1}^{n} c_{k}^{w_{k}}\right) ; \wedge_{k=1}^{n} w_{\tilde{a}_{k}}, \vee_{k=1}^{n} u_{\tilde{a}_{k}}, \vee_{k=1}^{n} y_{\tilde{a}_{k}}\right\rangle
\end{aligned}
$$

which is summarized as in Theorem 3.4.

Theorem 3.4 Let $\tilde{a}_{j}=\left\langle\left(a_{j}, b_{j}, c_{j}\right) ; w_{\tilde{a}_{j}}, u_{\tilde{a}_{j}}, y_{\tilde{a}_{j}}\right\rangle \in \Delta(j \in I)$. Then SVTrN ordered weighted geometric operator denoted by $G_{\text {ogo }}$, is defined as;

$$
G_{\text {ogo }}: \Delta^{n} \rightarrow \Delta, G_{\text {ogo }}\left(\tilde{a}_{1}, \tilde{a}_{2}, \ldots, \tilde{a}_{n}\right)=\left\langle\left(\prod_{k=1}^{n} a_{k}^{w_{k}}, \prod_{k=1}^{n} b_{k}^{w_{k}}, \prod_{k=1}^{n} c_{k}^{w_{k}}\right) ; \bigwedge_{k=1}^{n} w_{\tilde{a}_{k}}, \bigvee_{k=1}^{n} u_{\tilde{a}_{k}}, \bigvee_{k=1}^{n} y_{\tilde{a}_{k}}\right\rangle
$$

where $w_{k} \in[0,1], \sum_{k=1}^{n} w_{k}=1 ; \quad \tilde{b}_{k}=\left\langle\left(a_{k}, b_{k}, c_{k}\right) ; w_{\tilde{a}_{k}}, u_{\tilde{a}_{k}}, y_{\tilde{a}_{k}}\right\rangle$ is the $k$-th largest of the $n$ neutrosophic sets $\tilde{a}_{j}\left(j \in I_{n}\right)$ which is determined through using some ranking method in Definition 2.8. 
Proof The proof can be made by using mathematical induction on $n$ as;

for $n=2$, we have

$$
G_{\text {ogo }}\left(\tilde{a}_{1}, \tilde{a}_{2}\right)=\left\langle\left(\prod_{j=1}^{2} a_{j}^{w_{j}}, \prod_{j=1}^{2} b_{j}^{w_{j}}, \prod_{j=1}^{2} c_{j}^{w_{j}}\right) ; \bigwedge_{j=1}^{2} w_{\tilde{a_{j}}}, \bigvee_{j=1}^{2} u_{\tilde{a_{j}}}, \bigvee_{j=1}^{2} y_{\tilde{a_{j}}}\right\rangle
$$

If holds for $n=k$, that is

$$
G_{\text {ogo }}\left(\tilde{a}_{1}, \tilde{a}_{2}, \ldots, \tilde{a}_{k}\right)=\left\langle\left(\prod_{j=1}^{k} a_{j}^{w_{j}}, \prod_{j=1}^{k} w_{j} b_{j}^{w_{j}}, \prod_{j=1}^{k} c_{j}^{w_{j}}\right) ; \bigwedge_{j=1}^{k} w_{\tilde{a}_{j}}, \bigvee_{j=1}^{k} u_{\tilde{a}_{j}}, \bigvee_{j=1}^{k} y_{\tilde{a}_{j}}\right\rangle
$$

then, when $n=k+1$, by the operational laws in Definition 2.6, I have

$$
\begin{aligned}
G_{\text {ogo }}\left(\tilde{a}_{1}, \tilde{a}_{2}, \ldots, \tilde{a}_{k}, \tilde{a}_{k+1}\right)= & \left\langle\left(\prod_{j=1}^{k} a_{j}^{w_{j}}, \prod_{j=1}^{k} b_{j}^{w_{j}}, \prod_{j=1}^{k} c_{j}^{w_{j}}\right) ; \bigwedge_{j=1}^{k} w_{\tilde{a}_{j}}, \bigvee_{j=1}^{k} u_{\tilde{a}_{j}}, \bigvee_{j=1}^{k} y_{\tilde{a}_{j}}\right\rangle \\
& \times\left\langle\left(a_{k+1}^{w_{k+1}}, b_{k+1}^{w_{k+1}}, c_{k+1}^{w_{k+1}}\right) ; w_{\tilde{a}_{k+1}}, u_{\tilde{a}_{k+1}}, y_{\tilde{a}_{k+1}}\right\rangle \\
= & \left\langle\left(\prod_{j=1}^{k+1} a_{j}^{w_{j}}, \prod_{j=1}^{k+1} b_{j}^{w_{j}}, \prod_{j=1}^{k+1} c_{j}^{w_{j}}\right) ; \bigwedge_{j=1}^{k+1} w_{\tilde{a}_{j}}, \bigvee_{j=1}^{k+1} u_{\tilde{a}_{j}}, \bigvee_{j=1}^{k+1} y_{\tilde{a}_{j}}\right\rangle
\end{aligned}
$$

therefore proof is valid.

Now, we give an example (is adapted from [14].)

Example 3.5 There are four experts who are invited to evaluate some enterprise. Their evaluations are expressed with the single valued neutrosophic sets

$$
\begin{aligned}
& \tilde{a}_{1}=\langle(0.123,0.234,0.325) ; 0.4,0.5,0.7\rangle, \\
& \tilde{a}_{2}=\langle(0.234,0.354,0.451) ; 0.3,0.6,0.6\rangle, \\
& \tilde{a}_{3}=\langle(0.125,0.365,0.465) ; 0.2,0.7,0.5\rangle, \\
& \tilde{a}_{4}=\langle(0.215,0.345,0.435) ; 0.1,0.8,0.4\rangle,
\end{aligned}
$$

respectively. To eliminate effect of individual bias on comprehensive evaluation, the unduly high evaluation and the unduly low evaluation are punished through giving a smaller weight. Assume that the position weight vector is $w=(0.15,0.35,0.35,0.15)$. Compute the comprehensive evaluation of the four experts on the enterprise though using the neutrosophic ordered weighted averaging operator.

Solving According to Eq. (3.2), the scores of the neutrosophic sets $\tilde{a}_{j}(j=1,2,3,4)$ are obtained as follows:

$$
\begin{aligned}
& S\left(\tilde{a}_{1}\right)=\frac{1}{8}[0.123+0.234+0.325]=0.102 \times(2+0.4-0.5-0.7), \\
& S\left(\tilde{a}_{2}\right)=\frac{1}{8}[0.234+0.354+0.451]=0.143 \times(2+0.3-0.6-0.6), \\
& S\left(\tilde{a}_{3}\right)=\frac{1}{8}[0.125+0.365+0.465]=0.119 \times(2+0.2-0.7-0.5), \\
& S\left(\tilde{a}_{4}\right)=\frac{1}{8}[0.215+0.345+0.435]=0.112 \times(2+0.1-0.8-0.4),
\end{aligned}
$$

respectively. It is obvious that $S\left(\tilde{a}_{2}\right)>S\left(\tilde{a}_{3}\right)>S\left(\tilde{a}_{4}\right)>S\left(\tilde{a}_{1}\right)$. Hence according to the above scoring function ranking method, its follows that $\tilde{a}_{2}>\tilde{a}_{3}>\tilde{a}_{4}>\tilde{a}_{1}$. Hence, we have:

$$
\begin{aligned}
& \tilde{b}_{1}=\tilde{a}_{2}=\langle(0.234,0.354,0.451) ; 0.3,0.6,0.6\rangle, \\
& \tilde{b}_{2}=\tilde{a}_{3}=\langle(0.125,0.365,0.465) ; 0.2,0.7,0.5\rangle, \\
& \tilde{b}_{3}=\tilde{a}_{4}=\langle(0.215,0.345,0.435) ; 0.1,0.8,0.4\rangle,
\end{aligned}
$$




$$
\tilde{b}_{4}=\tilde{a}_{1}=\langle(0.123,0.234,0.325) ; 0.4,0.5,0.7\rangle
$$

Using Eq. (2), we obtain:

$$
\begin{aligned}
G_{\text {ogo }}\left(\tilde{a}_{1}, \tilde{a}_{2}, \tilde{a}_{3}, \tilde{a}_{4}\right)= & \left\langle\left( 0.234^{0.15} \times 0.125^{0.35} \times 0.215^{0.35} \times 0.123^{0.15}\right.\right. \\
& 0.354^{0.15} \times 0.365^{0.35} \times 0.345^{0.35} \times 0.234^{0.15} \\
& \left.\left.0.451^{0.15} \times 0.465^{0.35} \times 0.435^{0.35} \times 0.325^{0.15}\right) ; 0.1,0.8,0.7\right\rangle \\
= & \langle(0.166,0.333,0.429) ; 0.1,0.8,0.7\rangle
\end{aligned}
$$

Definition 3.6 Let $\tilde{a}_{j}=\left\langle\left(a_{j}, b_{j}, c_{j}\right) ; w_{\tilde{a}_{j}}, u_{\tilde{a}_{j}}, y_{\tilde{a}_{j}}\right\rangle \in \Delta(j \in I)$. Then $S V \operatorname{Tr} N$ ordered hybrid weighted geometric operator denoted by $G_{\text {hgo }}$, is defined as;

$$
G_{h g o}: \Delta^{n} \rightarrow \Delta, \quad G_{\text {ogo }}\left(\tilde{a}_{1}, \tilde{a}_{2}, \ldots, \tilde{a}_{n}\right)=\prod_{k=1}^{n} \hat{b}_{k}^{w_{k}}
$$

where $w=\left(w_{1}, w_{2}, \ldots, w_{n}\right)^{T} . w_{j} \in[0,1]$ and $\sum_{j=1}^{n} w_{j}=1$ is a weight vector associated with the mapping $G_{h g o}, a_{j} \in \Delta$ a weight with $n \omega\left(j \in I_{n}\right)$ is denoted by $\tilde{A}_{j}$ i.e., $\tilde{A}_{j}=n \omega \tilde{a}_{j}$, here $n$ is regarded as a balance factor $\omega=\left(\omega_{1}, \omega_{2}, \ldots, \omega_{n}\right)^{T}$ is a weight vector of the $a_{j} \in \Delta\left(j \in I_{n}\right) ; \hat{b}_{k}$ is the $k$-th largest of the $n S V T r N$ numbers $\tilde{A}_{j} \in \Delta\left(j \in I_{n}\right)$ which are determined through using some ranking method such as the above scoring function ranking method.

Note that if $\omega=(1 / n, 1 / n, \ldots, 1 / n)^{T}$, then $G_{h g o}$ degenerates to the $G_{o g o}$.

Example $3.7 \tilde{a}_{1}, \tilde{a}_{2}, \tilde{a}_{3} \in \Delta$. Their evaluations are expressed with the $G_{g o}$.

$$
\begin{aligned}
& \tilde{a}_{1}=\langle(0.123,0.234,0.325) ; 0.4,0.5,0.7\rangle, \\
& \tilde{a}_{2}=\langle(0.234,0.354,0.451) ; 0.3,0.6,0.6\rangle, \\
& \tilde{a}_{3}=\langle(0.125,0.365,0.465) ; 0.2,0.7,0.5\rangle, \\
& \tilde{a}_{4}=\langle(0.215,0.345,0.435) ; 0.1,0.8,0.4\rangle
\end{aligned}
$$

respectively. Assume that the weight vector of the three experts is $\omega=(0.2,0.3,0.3,0.2)^{T}$ and the position weight vector is $w=(0.4,0.1,0.1,0.4)^{T}$. Compute the comprehensive evaluation of the three experts on the decision alternative through using the $G_{\text {hgo }}$.

Solving

$$
\begin{aligned}
& \tilde{A}_{1}=4 \times 0.2 \times \tilde{a}_{1}=4 \times 0.2 \times\langle(0.123,0.234,0.325) ; 0.4,0.5,0.7\rangle=\langle(0.098,0.187,0.260) ; 0.4,0.5,0.7\rangle \\
& \tilde{A}_{2}=4 \times 0.3 \times \tilde{a}_{2}=4 \times 0.3 \times\langle(0.234,0.354,0.451) ; 0.3,0.6,0.6\rangle=\langle(0.281,0.425,0.541) ; 0.3,0.6,0.6\rangle \\
& \tilde{A}_{3}=4 \times 0.3 \times \tilde{a}_{3}=4 \times 0.3 \times\langle(0.125,0.365,0.465) ; 0.2,0.7,0.5\rangle=\langle(0.150,0.438,0.558) ; 0.2,0.7,0.5\rangle \\
& \tilde{A}_{4}=4 \times 0.2 \times \tilde{a}_{4}=4 \times 0.2 \times\langle(0.215,0.345,0.435) ; 0.1,0.8,0.4\rangle=\langle(0.172,0.276,0.348) ; 0.1,0.8,0.4\rangle
\end{aligned}
$$

we obtain the scores of the SVTrN-numbers $\tilde{A}_{j}(j=1,2,3)$ as follows:

$$
\begin{aligned}
S\left(\tilde{A}_{1}\right) & =\frac{1}{8}[0.098+0.187+0.260] \times(2+0.4-0.5-0.7)=0.082, \\
S\left(\tilde{A}_{2}\right) & =\frac{1}{8}[0.281+0.425+0.541] \times(2+0.3-0.6-0.6)=0.171 \\
S\left(\tilde{A}_{3}\right) & =\frac{1}{8}[0.150+0.438+0.558] \times(2+0.2-0.7-0.5)=0.143, \\
S\left(\tilde{A}_{4}\right) & =\frac{1}{8}[0.172+0.276+0.348] \times(2+0.1-0.8-0.4)=0.090 .
\end{aligned}
$$


Obviously, $S\left(\tilde{A}_{2}\right)>S\left(\tilde{A}_{3}\right)>S\left(\tilde{A}_{1}\right)>S\left(\tilde{A}_{4}\right)$. Thereby, according to the above scoring function ranking method, we have

$$
\begin{aligned}
& \hat{b}_{1}=\tilde{A}_{2}=\langle(0.281,0.425,0.541) ; 0.3,0.6,0.6\rangle \\
& \hat{b}_{2}=\tilde{A}_{3}=\langle(0.150,0.438,0.558) ; 0.2,0.7,0.5\rangle \\
& \hat{b}_{3}=\tilde{A}_{4}=\langle(0.172,0.276,0.348) ; 0.1,0.8,0.4\rangle \\
& \hat{b}_{4}=\tilde{A}_{1}=\langle(0.098,0.187,0.260) ; 0.4,0.5,0.7\rangle
\end{aligned}
$$

It follows from (3.2) that

$$
\begin{aligned}
G_{h g o}\left(\tilde{a}_{1}, \tilde{a}_{2}, \tilde{a}_{3}, \tilde{a}_{4}\right)= & \langle(0.281 \times 0.4+0.150 \times 0.1+0.172 \times 0.1+0.098 \times 0.4, \\
& 0.425 \times 0.4+0.438 \times 0.1+0.276 \times 0.1+0.187 \times 0.4, \\
= & 0.541 \times 0.4+0.558 \times 0.1+0.348 \times 0.1+0.260 \times 0.4) ; 0.1,0.8,0.7\rangle \\
= & \langle(0.184,0.316,0.411) ; 0.1,0.8,0.7\rangle
\end{aligned}
$$

Theorem 3.8 Let $\tilde{a}_{j}=\left\langle\left(a_{j}, b_{j}, c_{j}\right) ; w_{\tilde{a}_{j}}, u_{\tilde{a}_{j}}, y_{\tilde{a}_{j}}\right\rangle \in \Delta\left(j \in I_{n}\right), w=\left(w_{1}, w_{2}, \ldots, w_{n}\right)^{T}$ be a weight vector of

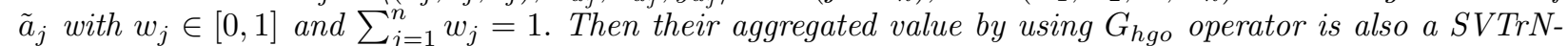
number and

$$
G_{h g o}: \Delta^{n} \rightarrow \Delta, G_{h g o}\left(\tilde{a}_{1}, \tilde{a}_{2}, \ldots, \tilde{a}_{n}\right)=\left\langle\left(\prod_{k=1}^{n} a_{k}^{w_{k}}, \prod_{k=1}^{n} b_{k}^{w_{k}}, \prod_{k=1}^{n} c_{k}^{w_{k}}\right) ; \bigwedge_{k=1}^{n} w_{\tilde{a}_{k}}, \bigvee_{k=1}^{n} u_{\tilde{a}_{k}}, \bigvee_{k=1}^{n} y_{\tilde{a}_{k}}\right\rangle
$$

where $\hat{b}_{k}=\left\langle\left(a_{k}, b_{k}, c_{k}\right) ; w_{\tilde{a}_{k}}, u_{\tilde{a}_{k}}, y_{\tilde{a}_{k}}\right\rangle$ is the $\mathrm{k}$-th largest of the $n S V \operatorname{Tr} N$-numbers $\hat{A}_{j}=n \omega_{j} \tilde{a}_{j}\left(j \in I_{n}\right)$ which is determined through using some ranking method such as the above scoring function ranking method.

Proof Theorem 3.8 can be proven in a similar way to that of Theorem 3.4 (omitted).

\section{Multi-criteria decision making based on SVTrN-numbers}

In this section, we define a multi-criteria decision making method, so called SVTrN-multi-criteria decisionmaking method, by using the $G_{h g o}$ operator. Some of it is quoted from application in [10, 14, 17, 18, 37].

There is a panel with four possible alternatives to invest the money (adapted from [18]): (1) $x_{1}$ is a car company; (2) $x_{2}$ is a food company; (3) $x_{3}$ is a computer company; (4) $x_{4}$ is a television company. The investment company must take a decision according to the following three criteria: (1) $u_{1}$ is the risk analysis; (2) $u_{2}$ is the growth analysis; (3) $u_{3}$ is the environmental impact analysis; (4) $u_{4}$ social political impact analysis. The four possible alternatives are to be evaluated under the above three criteria by corresponding to linguistic values of SVTrN-numbers for linguistic terms (adapted from [37]), as shown in Table 1.

\begin{tabular}{ll}
\hline Linguistic terms & Linguistic values of SVTrN-numbers \\
\hline Absolutely low & $\langle(0.1,0.2,0.3) ; 0.1,0.2,0.3\rangle$ \\
Low & $\langle(0.2,0.3,0.4) ; 0.2,0.3,0.4\rangle$ \\
Fairly low & $\langle(0.3,0.4,0.5) ; 0.3,0.4,0.5\rangle$ \\
Medium & $\langle(0.4,0.5,0.6) ; 0.4,0.5,0.6\rangle$ \\
Fairly high & $\langle(0.5,0.6,0.7) ; 0.5,0.6,0.7\rangle$ \\
High & $\langle(0.6,0.7,0.8) ; 0.6,0.7,0.8\rangle$ \\
Absolutely high & $\langle(0.7,0.8,0.9) ; 0.7,0.8,0.9\rangle$ \\
\hline
\end{tabular}

Table 1: SVTrN-numbers for linguistic terms 
Definition 4.1 Let $X=\left(x_{1}, x_{2}, \ldots, x_{m}\right)$ be a set of alternatives, $U=\left(u_{1}, u_{2}, \ldots, u_{n}\right)$ be the set of attributes. If $\tilde{a}_{i j}=\left\langle\left(a_{i j}, b_{i j}, c_{i j}\right) ; w_{i j}, u_{i j}, y_{i j}\right\rangle \in \Delta$, then

$$
\left[\tilde{a}_{i j}\right]_{m \times n}=\begin{gathered}
x_{1} \\
x_{2} \\
\vdots \\
x_{m}
\end{gathered}\left(\begin{array}{cccc}
\tilde{a}_{11} & u_{22} & \cdots & u_{n} \\
\tilde{a}_{21} & \tilde{a}_{22} & \cdots & \tilde{a}_{1 n} \\
\vdots & \vdots & \vdots & \vdots \\
\tilde{a}_{m 1} & \tilde{a}_{m 2} & \cdots & \tilde{a}_{m n}
\end{array}\right)
$$

is called an SVTrN-multi-criteria decision-making matrix of the decision maker.

Now, we can give an algorithm of the SVTrN-multi-criteria decision-making method as follows;

\section{Algorithm:}

Step 1. Construct the decision-making matrix $\left[\tilde{a}_{i j}\right]_{m \times n}$ for decision;

Step 2. Compute the SVTrN-numbers $\tilde{A}_{i j}=n \omega_{i} \tilde{a}_{i j}\left(i \in I_{m} ; j \in I_{n}\right)$ and write the decision-making matrix $\left[\tilde{A}_{i j}\right]_{m \times n}$

Step 3. Obtain the scores of the SVTrN-numbers $\tilde{A}_{i j}\left(i \in I_{m} ; j \in I_{n}\right)$;

Step 4. Rank all SVTrN-numbers $\tilde{A}_{i j}\left(i \in I_{m} ; j \in I_{n}\right)$ by using the ranking method of SVTrN-numbers and determine the SVTrN-numbers $\left[b_{i}\right]_{1 \times n}=\tilde{b}_{i k}\left(i \in I_{m} ; k \in I_{n}\right)$ where $\tilde{b}_{i k}$ is k-th largest of $\tilde{A}_{i j}$ for $j \in I_{n}$;

Step 5. Give the decision matrix $\left[b_{i}\right]_{1 \times n}$ for $i=1,2,3,4$;

Step 6. Compute $G_{h g o}\left(\tilde{b}_{i 1}, \tilde{b}_{i 2}, \ldots, \tilde{b}_{i n}\right)$ for $i \in I_{m}$;

Step 7. Rank all alternatives $x_{i}$ by using the ranking method of SVTrN-numbers and determine the best alternative.

\section{Application}

In this section, we give an application for the SVTrN-multi-criteria decision-making method, by using the $G_{h g o}$ operator. Some of it is quoted from application in [10, 14, 18, 37].

Example 5.1 Let us consider the decision-making problem adapted from [41]. There is an investment company, which wants to invest a sum of money in the best option. There is a panel with the set of the four alternatives is denoted by $X=\left\{x_{1}=\right.$ car company, $x_{2}=$ food company, $x_{3}=$ computer company, $x_{4}=$ television company to invest the money. The investment company must take a decision according to the set of the four attributes is denoted by $U=\left\{u_{1}=\right.$ risk analysis, $u_{2}=$ growth analysis, $u_{3}=$ environmental impact analysis, $u_{4}=$ social political impact analysis $\}$. Then, the weight vector of the attributes is $\omega=(0.1,0.2,0.3,0.4)^{T}$ and the position weight vector is $w=(0.24,0.26,0.26,0.24)^{T}$ by using the weight determination based on the normal distribution. For the evaluation of an alternative $x_{i}(i=1,2,3,4)$ with respect to a criterion $u_{j}$ $(j=1,2,3,4)$, it is obtained from the questionnaire of a domain expert. Then,

Step 1. The decision maker construct the decision matrix $\left[\tilde{a}_{i j}\right]_{4 x 4}$ as follows:

$$
u_{1}
$$$$
\begin{aligned}
& x_{1} \\
& x_{2} \\
& x_{3} \\
& x_{4}
\end{aligned} \quad \begin{aligned}
& (0.1,0.2,0.3) ; 0.1,0.2,0.3 \\
& (0.3,0.6,0.9) ; 0.3,0.6,0.9 \\
& (0.6,0.3,0.4) ; 0.2,0.3,0.4 \\
& (0.8) ; 0.6,0.7,0.8
\end{aligned}
$$

$$
u_{2}
$$

$(0.3,0.4,0.5) ; 0.3,0.4,0.5$

$(0.1,0.6,0.9) ; 0.1,0.6,0.9$

$(0.5,0.6,0.7) ; 0.5,0.6,0.7$

$(0.2,0.3,0.8) ; 0.2,0.3,0.8$
$(0.6,0.7,0.8) ; 0.6,0.7,0.8$ $(0.4,0.5,0.6) ; 0.4,0.5,0.6$

$(0.7,0.8,0.9) ; 0.7,0.8,0.9$

$(0.2,0.7,0.8) ; 0.2,0.7,0.8$ $u_{4}$

$(0.1,0.3,0.9) ; 0.1,0.3,0.9$

$(0.1,0.6,0.9) ; 0.1,0.6,0.9$

$(0.2,0.4,0.8) ; 0.2,0.4,0.8$

$(0.2,0.7,0.8) ; 0.2,0.7,0.8)$ 
Step 2. Compute $\tilde{A}_{i j}=n \omega_{i} \tilde{a}_{i j}(i=1,2,3,4 ; j=1,2,3,4)$ as follows:

$$
\begin{aligned}
\tilde{A}_{11} & =4 \times 0.1 \times \tilde{a}_{11} \\
& =\left\langle\left(0.1^{0.4}, 0.2^{0.4}, 0.3^{0.4}\right) ; 0.1,0.2,0.3\right\rangle \\
& =\langle(0.398,0.525,0.618) ; 0.1,0.2,0.3\rangle
\end{aligned}
$$

Likewise, we can obtain other SVTrN-numbers $\tilde{A}_{i j}=n \omega_{i} \tilde{a}_{i j}(i=1,2,3,4 ; j=1,2,3,4)$ which are given by the SVTrN-decision matrix $\left[\tilde{A}_{i j}\right]_{4 \times 4}$ as follows:

$\left[\tilde{A}_{i j}\right]_{4 \times 4}=$

$x_{1}$
$x_{2}$
$x_{3}$
$x_{4}$$\quad\left(\begin{array}{l}(0.398,0.525,0.618) ; 0.1,0.2,0.3 \\ (0.525,0.618,0.693) ; 0.2,0.3,0.4 \\ (0.815,0.867,0.915) ; 0.6,0.7,0.8\end{array}\right.$

$(0.382,0.480,0.574) ; 0.3,0.4,0.5$ $(0.158,0.665,0.919) ; 0.1,0.6,0.9$ $(0.574,0.665,0.754) ; 0.5,0.6,0.7$

$(0.276,0.382,0.837) ; 0.2,0.3,0.8$ $u_{3}$

$(0.542,0.652,0.765) ; 0.6,0.7,0.8$ $(0.333,0.435,0.542) ; 0.4,0.5,0.6$ $(0.652,0.765,0.881) ; 0.7,0.8,0.9$ $(0.145,0.652,0.765) ; 0.2,0.7,0.8$
$(0.025,0.146,0.845) ; 0.1,0.3,0.9$ $(0.025,0.442,0.845) ; 0.1,0.6,0.9$ $(0.076,0.231,0.700) ; 0.2,0.4,0.8$ $(0.076,0.231,0.700) ; 0.2,0.4,0.8$
$(0.076,0.565,0.700) ; 0.2,0.7,0.8$

Step 3. We can obtain the scores of the SVTrN-numbers $\tilde{A}_{i j}$ of the alternatives $x_{j}(j=1,2,3,4)$ on the four attributes $u_{i}(i=1,2,3,4)$ as follows:

$$
\begin{array}{llll}
S\left(\tilde{A}_{11}\right)=0.308 & S\left(\tilde{A}_{12}\right)=0.251 & S\left(\tilde{A}_{13}\right)=0.269 & S\left(\tilde{A}_{14}\right)=0.114 \\
S\left(\tilde{A}_{21}\right)=0.239 & S\left(\tilde{A}_{22}\right)=0.131 & S\left(\tilde{A}_{23}\right)=0.213 & S\left(\tilde{A}_{24}\right)=0.098 \\
S\left(\tilde{A}_{31}\right)=0.344 & S\left(\tilde{A}_{32}\right)=0.299 & S\left(\tilde{A}_{33}\right)=0.137 & S\left(\tilde{A}_{34}\right)=0.126 \\
S\left(\tilde{A}_{41}\right)=0.357 & S\left(\tilde{A}_{42}\right)=0.205 & S\left(\tilde{A}_{43}\right)=0.287 & S\left(\tilde{A}_{44}\right)=0.117
\end{array}
$$

Step 4. The ranking order of all SVTrN-numbers $\tilde{A}_{i j}(i=1,2,3,4 ; j=1,2,3,4)$ as follows;

$$
\begin{aligned}
\tilde{A}_{11}>\tilde{A}_{13}>\tilde{A}_{12}>\tilde{A}_{14} \\
\tilde{A}_{21}>\tilde{A}_{23}>\tilde{A}_{22}>\tilde{A}_{24} \\
\tilde{A}_{31}>\tilde{A}_{32}>\tilde{A}_{33}>\tilde{A}_{34} \\
\tilde{A}_{41}>\tilde{A}_{43}>\tilde{A}_{42}>\tilde{A}_{44}
\end{aligned}
$$

Thus, we have:

$$
\begin{aligned}
& \tilde{b}_{11}=\tilde{A}_{11}, \tilde{b}_{12}=\tilde{A}_{13}, \tilde{b}_{13}=\tilde{A}_{12}, \tilde{b}_{14}=\tilde{A}_{14} \\
& \tilde{b}_{21}=\tilde{A}_{21}, \tilde{b}_{22}=\tilde{A}_{23}, \quad \tilde{b}_{23}=\tilde{A}_{22}, \tilde{b}_{24}=\tilde{A}_{24} \\
& \tilde{b}_{31}=\tilde{A}_{31}, \tilde{b}_{32}=\tilde{A}_{32}, \quad \tilde{b}_{33}=\tilde{A}_{33}, \quad \tilde{b}_{34}=\tilde{A}_{34} \\
& \tilde{b}_{41}=\tilde{A}_{41}, \tilde{b}_{42}=\tilde{A}_{43}, \quad \tilde{b}_{43}=\tilde{A}_{42}, \tilde{b}_{44}=\tilde{A}_{44}
\end{aligned}
$$

Step 5. The decision matrix $\left[b_{i}\right]_{1 \times n}$ for $i=1,2,3,4$ are given by;

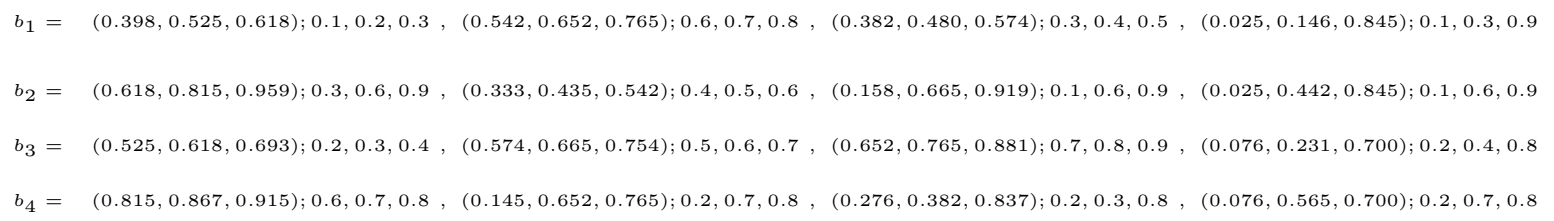

Step 6. We can calculate the SVTrN-numbers $G_{h g o}\left(b_{i}\right)=G_{h g o}\left(\tilde{b}_{i 1}, \tilde{b}_{i 2}, \tilde{b}_{i 3}, \tilde{b}_{i 4}\right)$ for $i=1,2,3,4$ as follows:

$$
\begin{aligned}
G_{\text {hgo }}\left(b_{1}\right)= & G_{\text {hgo }}\left(\tilde{b}_{11}, \tilde{b}_{12}, \tilde{b}_{13}, \tilde{b}_{14}\right) \\
= & \left\langle\left( 0.398^{0.24} \times 0.618^{0.26} \times 0.525^{0.26} \times 0.815^{0.24},\right.\right. \\
& 0.525^{0.24} \times 0.815^{0.26} \times 0.618^{0.26} \times 0.867^{0.24}, \\
& \left.\left.0.618^{0.24} \times 0.959^{0.26} \times 0.693^{0.26} \times 0.915^{0.24}\right) ; 0.1,0.7,0.9\right\rangle \\
= & \langle(0.570,0.693,0.784) ; 0.1,0.7,0.9\rangle
\end{aligned}
$$




$$
\begin{aligned}
G_{\text {hgo }}\left(b_{2}\right)= & G_{\text {hgo }}\left(\tilde{b}_{11}, \tilde{b}_{12}, \tilde{b}_{13}, \tilde{b}_{14}\right) \\
= & \left\langle\left( 0.542^{0.24} \times 0.333^{0.26} \times 0.574^{0.26} \times 0.145^{0.24},\right.\right. \\
& 0.652^{0.24} \times 0.435^{0.26} \times 0.665^{0.26} \times 0.652^{0.24}, \\
& \left.\left.0.765^{0.24} \times 0.542^{0.26} \times 0.754^{0.26} \times 0.765^{0.24}\right) ; 0.2,0.7,0.8\right\rangle \\
= & \langle(0.312,0.538,0.762) ; 0.2,0.7,0.8\rangle \\
G_{\text {hgo }}\left(b_{3}\right)= & G_{\text {hgo }}\left(\tilde{b}_{11}, \tilde{b}_{12}, \tilde{b}_{13}, \tilde{b}_{14}\right) \\
= & \left\langle\left( 0.382^{0.24} \times 0.158^{0.26} \times 0.652^{0.26} \times 0.276^{0.24},\right.\right. \\
& 0.480^{0.24} \times 0.665^{0.26} \times 0.765^{0.26} \times 0.382^{0.24}, \\
& \left.\left.0.574^{0.24} \times 0.919^{0.26} \times 0.881^{0.26} \times 0.837^{0.24}\right) ; 0.1,0.8,0.9\right\rangle \\
= & \langle(0.365,0.612,0.726) ; 0.1,0.8,0.9\rangle \\
G_{\text {hgo }}\left(b_{4}\right)= & G_{h g o}\left(\tilde{b}_{11}, \tilde{b}_{12}, \tilde{b}_{13}, \tilde{b}_{14}\right) \\
= & \left\langle\left( 0.025^{0.24} \times 0.025^{0.26} \times 0.076^{0.26} \times 0.076^{0.24},\right.\right. \\
& 0.146^{0.24} \times 0.442^{0.26} \times 0.231^{0.26} \times 0.565^{0.24}, \\
& \left.\left.0.845^{0.24} \times 0.845^{0.26} \times 0.700^{0.26} \times 0.700^{0.24}\right) ; 0.1,0.7,0.9\right\rangle \\
= & \langle(0.044,0.303,0.769) ; 0.1,0.7,0.9\rangle
\end{aligned}
$$

Step 7. The scores of $G_{h g o}\left(\tilde{b}_{i}\right)$ for $i=1,2,3,4$ can be obtained as follows:

$$
\begin{aligned}
& S\left(G_{\text {hgo }}\left(b_{1}\right)\right)=0.128 \\
& S\left(G_{\text {hgo }}\left(b_{2}\right)\right)=0.121 \\
& S\left(G_{\text {hgo }}\left(b_{3}\right)\right)=0.106 \\
& S\left(G_{\text {hgo }}\left(b_{4}\right)\right)=0.070
\end{aligned}
$$

It is obvious that

$$
G_{\text {hgo }}\left(b_{1}\right)>G_{\text {hgo }}\left(b_{2}\right)>G_{\text {hgo }}\left(b_{3}\right)>G_{\text {hgo }}\left(b_{4}\right)
$$

Therefore, the ranking order of the alternatives $x_{j}(j=1,2,3,4)$ is generated as follows:

$$
x_{1} \succ x_{2} \succ x_{3} \succ x_{4}
$$

The best supplier for the enterprise is $x_{1}$.

\section{Conclusion}

This paper proposes three geometric operator is called SVTrN weighted geometric operator, SVTrN ordered weighted geometric operator, SVTrN ordered hybrid weighted geometric operator. Then, a approach is developed to solve multi-criteria decision making problems. It is easily seen that the proposed approach can be extended to solve more general multi-criteria decision making problems in a straightforward manner. Due to the fact that a SVTrN-number is a generalization of a triangular fuzzy number and triangular intuitionistic fuzzy number, the other existing approaches of triangular fuzzy number and triangular intuitionistic fuzzy number may be extended to SVTrN-numbers. Therefore,

1. More effective approaches for SVTrN-numbers,

2. How to determine the weight vectors for SVTrN-numbers,

3. An approach of multi-criteria decision-making with weights expressed by single valued neutrosophic sets,

will be investigated in the near future. 


\section{References}

[1] K. Atanassov, Intuitionistic fuzzy sets, Fuzzy Sets and Systems, 20 (1986) 87-96.

[2] S. Broumi, F. Smarandache, Single valued neutrosophic trapezoid linguistic aggregation operators based multi-attribute decision making, Bulletin of Pure and Applied Sciences Mathematics and Statistics, DOI : 10.5958/2320-3226.2014.00006.X.

[3] S. Broumi, I. Deli, F. Smarandache, Relations on Interval Valued Neutrosophic Soft Sets, Journal of New Results in Science 5 (2014) 01-20.

[4] S. Broumi, I. Deli, F. Smarandache, Distance and Similarity Measures of Interval Neutrosophic Soft Sets, Critical Review, Center for Mathematics of Uncertainty, Creighton University, USA, 8 (2014) $14-31$.

[5] S. Broumi, I. Deli, F. Smarandache, Interval valued neutrosophic parameterized soft set theory and its decision making, Journal of New Results in Science 7 (2014) 58-71.

[6] S. Broumi and . Deli, Correlation measure for neutrosophic Refined sets and its application n medical Diagnosis, Palestine journal of mathematics, 3(1) (2014), 11-19.

[7] Y. Guo, A. Şengür, A novel image segmentation algorithm based on neutrosophic similarity clustering, Applied Soft Computing 25 (2014) 391-398.

[8] N. Cağman, I. Deli, Means of FP-Soft Sets and its Applications, Hacettepe Journal of Mathematics and Statistics, 41/5 (2012) 615-625.

[9] N. Cağman, I. Deli, Product of FP-Soft Sets and its Applications, Hacettepe Journal of Mathematics and Statistics 41/3 (2012) $365-374$.

[10] I. Deli, Y. Şubaş, Neutrosophic numbers and their applications to multicriteria decision making problem, (accepted).

[11] I. Deli and S. Broumi, Neutrosophic Soft Matrices and NSM-decision Making, Journal of Intelligent and Fuzzy Systems, Doi: 10.3233/IFS-141505.

[12] I. Deli, N. aman, Intuitionistic fuzzy parameterized soft set theory and its decision making, Applied Soft Computing 28 (2015) 109-113.

[13] I. Deli and S. Broumi, Neutrosophic soft relations and some properties, Annals of Fuzzy Mathematics and Informatics 9(1) ( 2015) 169-182.

[14] D. F. Li, Decision and Game Theory in Management With Intuitionistic Fuzzy Sets Studies in Fuzziness and Soft Computing Volume 308, springer, 2014.

[15] Y. He, H. Chen, L. Zhou, B. Han, Q. Zhao, J. Liu, Generalized intuitionistic fuzzy geometric interaction operators and their application to decision making, Expert Systems with Applications 41 (2014) 24842495.

[16] Y. He, H. Chen, L. Zhou, B. Han, Q. Zhao, J. Liu, Generalized intuitionistic fuzzy geometric interaction operators and their application to decision making, Expert Systems with Applications 41 (2014) 24842495.

[17] Y. He, H. Chen, L. Zhou, J. Liu, Z. Tao, Intuitionistic fuzzy geometric interaction averaging operators and their application to multi-criteria decision making, Information Sciences 259 (2014) 142-159.

[18] F. Herrera, E. H. Viedma, Linguistic decision analysis: steps for solving decision problems under linguistic information, Fuzzy Sets and Systems 115 (2000) 67-82.

[19] R. Krohling, A. Pacheco, A. Siviero, IF-TODIM: An intuitionistic fuzzy TODIM to multi-criteria decision making, Knowledge-Based Systems 53 (2013) 142-146.

[20] P. Liu, Y. Chu, Y. Li, Y. Chen, Some Generalized Neutrosophic Number Hamacher Aggregation Operators and Their Application to Group Decision Making, International Journal of Fuzzy Systems, 16/2 (2014) 242-255. 
[21] D. Li, The GOWA operator based approach to multiattribute decision making using intuitionistic fuzzy sets, Mathematical and Computer Modelling 53 (2011) 1182-1196.

[22] C. Liang, S. Zhao, J. Zhang, Aggregaton Operators on Trangular Intutonstc Fuzzy Numbers and its Applcaton to Mult-Crtera Decson Makng Problems, Foundations of Computing and Decision Sciences $39 / 3$ (2014) 189-208.

[23] J.J. Peng, J.Q. Wang, X.H. Wu, J. Wang, X.H. Chen, Multi-valued Neutrosophic Sets and Power Aggregation Operators with Their Applications in Multi-criteria Group Decision-making Problems, International Journal of Computational Intelligence Systems, 8/2 (2014) 345-363.

[24] F.Smarandache, "A Unifying Field in Logics. Neutrosophy: Neutrosophic Probability, Set and Logic". Rehoboth: American Research Press, 1998.

[25] F. Smarandache, Neutrosophic set, a generalisation of the intuitionistic fuzzy sets, Int. J. Pure Appl. Math. 24 (2005) 287-297.

[26] Z.Xu, R. R. Yager, Some geometric aggregation operators based on intuitionistic fuzzy sets, International Journal of General Systems, 35(4) (2006), 417433.

[27] S. Wan, J.Dong, Power Geometric Operators of Trapezoidal Intuitionistic Fuzzy Numbers and Application to Multi-attribute Group Decision Making, Applied Soft Computing Journal (2015), http://dx.doi.org/10.1016/j.asoc.2014.12.031.

[28] H. Wang, F. Y. Smarandache, Q. Zhang, R. Sunderraman, Single valued neutrosophic sets, Multispace and Multistructure 4 (2010) 410-413.

[29] J. Wang, R. Nie, H. Zhang, X. Chen, New operators on triangular intuitionistic fuzzy numbers and their applications in system fault analysis, Information Sciences 251 (2013) 79-95.

[30] S. P. Wan, Power average operators of trapezoidal intuitionistic fuzzy numbers and application to multi-attribute group decision making, Applied Mathematical Modelling 37 (2013) 4112-4126.

[31] G. Wei, Some induced geometric aggregation operators with intuitionistic fuzzy information and their application to group decision making, Applied Soft Computing 10 (2010) 423-431.

[32] J. Wu, Q.W. Cao Same families of geometric aggregation operators with intuitionistic trapezoidal fuzzy numbers, Applied Mathematical Modelling 37 (2013) 318-327.

[33] J. Wu, A SD-LITFOWA Operator And Topsis Based Approach For Magdm Problems Wth Intutonstc Trapezodal Fuzzy Numbers, Technological And Economic Development Of Economy 21/1 (2015) 28-47.

[34] Z. Xu, X. Cai, Group Decision Making with Incomplete Interval-Valued Intuitionistic Preference Relations, Group Decision and Negotiation (2015) 24(2), 193-215.

[35] S. Zeng, Some Intuitionistic Fuzzy Weighted Distance Measures and Their Application to Group Decision Making, Group Decision and Negotiation, (2013) 22(2), 281-298.

[36] Z. Xu, X. Cai, Group Decision Making with Incomplete Interval-Valued Intuitionistic Preference Relations, Group Decision and Negotiation (2015) 24(2), 193-215.

[37] J. Ye, Expected value method for intuitionistic trapezoidal fuzzy multicriteria decision-making problems, Expert Systems with Applications 38 (2011) 11730-11734.

[38] J. Ye, Single Valued Neutrosophic Cross-Entropy for Multicriteria Decision Making Problems, Applied Mathematical Modelling 38 (2014) 1170-1175

[39] J. Ye, Improved cosine similarity measures of simplified neutrosophic sets for medical diagnoses, Artificial Intelligence in Medicine (2014), http://dx.doi.org/10.1016/j.artmed.2014.12.007

[40] J. Ye, Vector Similarity Measures of Simplified Neutrosophic Sets and Their Application in Multicriteria Decision Making, International Journal of Fuzzy Systems, 16/2 (2014) 204-211.

[41] J. Ye, Trapezoidal neutrosophic set and its application to multiple attribute decision-making, Neural Comput. and Appl., DOI:10.1007/s00521-014-1787-6. 
[42] J. Ye, Single-Valued Neutrosophic Minimum Spanning Tree and Its Clustering Method, Journal of Intelligent Systems 23/3 (2014) 311324.

[43] J. Ye, Similarity measures between interval neutrosophic sets and their applications in multicriteria decision-making, Journal of Intelligent Fuzzy Systems 26 (2014) 165-172.

[44] L.A. Zadeh, Fuzzy Sets, Information and Control, 8, 338-353, 1965. 